East African Medical Journal Vol. 83 No. 5 May 2006

EARLY PERINATAL OUTCOME IN CASES DELIVERED THROUGH CAESARIAN SECTION FOLLOWING CLINICAL DIAGNOSIS OF SEVERE FOETAL DISTRESS AT KENYATTA NATIONAL HOSPITAL

S.K. Rotich, MBChB, Senior House Officer and Registrar, P.M. Ndavi, MBChB, MMed, MSc (Epid), DLSHTM, FHBR, Senior Lecturer and Honorary Consultant, Kenyatta National Hospital, R. Rukaria-Kaumbutho, MBChB, MMed, Lecturer, Department of Obstetrics and Gynaecology, College of Health Sciences, University of Nairobi, P.O. Box 19676-00202, Nairobi, Kenya, and Honorary Consultant Kenyatta National Hospital, C. Sekkade-Kigondu, PhD, Associate Professor, Department of Clinical Chemistry, College of Health Sciences, University of Nairobi, P.O. Box 19676-00202, Nairobi, Kenya

Request for reprints to: Dr. P.M. Ndavi, Department of Obstetrics and Gynaecology, College of Health Sciences, University of Nairobi, P.O. Box 19676-00202, Nairobi, Kenya

\title{
EARLY PERINATAL OUTCOME IN CASES DELIVERED THROUGH CAESARIAN SECTION FOLLOWING CLINICAL DIAGNOSIS OF SEVERE FOETAL DISTRESS AT KENYATTA NATIONAL HOSPITAL
}

\author{
S.K. ROTICH, M.P. NDAVI, R. RUKARIA-KAUMBUTHO and C.S. KIGONDU
}

\begin{abstract}
Objectives: To determine the value of clinical foetal distress in predicting early perinatal outcome. Design: Prospective cohort study.

Setting: Kenyatta National Hospital.

Subjects: Fifty eight newborns delivered via Caesarian section with a diagnosis of clinical foetal distress were compared with another group of 58 newborns delivered similarly, but without clinical foetal distress.

Results: Newborn acidemia was found in $71 \%$ of newborns with clinical foetal distress in contrast to $\mathbf{1 7 \%}$ in newborns without foetal distress. Low Apgar score at one minute was noted in about $59 \%$ of newborns with foetal distress compared with $31 \%$ in newborns without foetal distress. Similarly, 24.1\% of neonates with clinical foetal distress had low Apgar score at five minutes compared with $3.4 \%$ in those without foetal distress. Thirty one percent of newborns with clinical foetal distress were admitted to newborn unit for more than 24 hours due to respiratory distress or birth asphyxia compared to $17 \%$ of those without foetal distress. The incidence of morbidity and or mortality in newborns exposed to foetal distress was twice the one of newborns without foetal distress.

Conclusion: The results obtained in this study agreed with those who consider intrapartum passage of meconeum and abnormal foetal heart rate and rhythm to signify clinical foetal distress that carries bad prognostic outcome. These two parameters should still be used to indicate foetal distress which requires immediate institution of supportive therapy and immediate delivery.
\end{abstract}

\section{INTRODUCTION}

Foetal distress may be defined as a physiological state in which there is metabolic acidosis secondary to hypoxia. It is brought about by factors that cause umbilical cord compression or impair gaseous exchange between the placenta and maternal circulation. Clinically, it is characterised by abnormal foetal heart rate and rhythm, passage of meconeum into amniotic fluid, and decreased foetal movements.
When foetal distress occurs in the presence of meconeum in the amniotic fluid, the risk of newborn respiratory depression, morbidity and mortality are greater than if the meconeum is not present $(1,2)$.

A diagnosis of clinical foetal distress is usually made when meconeum staining of amniotic fluid is found with foetal tachycardia of more than 160 beats per minute or bradycardia of less than 110 beats per 
minute, and, more so if there is abnormal foetal heart rhythm $(3,4)$. A report of decreased foetal movements by the mother together with poor biophysical profile score of less than six are also considered as indicators of clinical foetal distress (5). When Cardiotocogram is in use, a finding of late deceleration and or loss of beat to beat variability are also indicative of foetal distress.

Meconeum staining of amniotic fluid may be graded by physical examination into grades 1,2 , and 3 , which correspond with light, moderate and thick meconeum staining. In grade 1 the stained amniotic fluid will appear yellow or light green and if placed in a glass test-tube the examiner will readily see light shone through it. In grade 2 the amniotic fluid will appear green and if placed in a glass test-tube and light shone through it will allow less light to pass through. Grade 3 meconeum stained amniotic fluid will be dark green to black, thick to particulate and tenacious, and will not allow light to shine through (opaque) when placed in the test-tube $(1,2)$.

Early perinatal outcome comprising of newborn asphyxia with low Apgar score at one and five minutes, cord blood acidaemia and features of cerebral irritation with increased neonatal morbidity and mortality have been associated with meconeum stained liquor grade 2 or more along with foetal heart rate and rhythm abnormalities (1,5-7). Umbilical cord blood gas and $\mathrm{pH}$ analyses are not routinely undertaken as part of evaluation of the newborn in Kenyatta National Hospital. Features that may indicate newborn asphyxia include Apgar score of five or less at one minute and low cord blood $\mathrm{pH}$ of less than 7.2. Sykes (2) reported that umbilical cord blood $\mathrm{pH}$ determination better reflects the infant's condition at birth than does the Apgar score. Neurological signs of central nervous system irritation such as cycling, fisting, starring and convulsion within 48 hours of birth may also follow severe birth asphyxia. Severe asphyxia secondary to foetal distress is one of the causes of cerebral palsy, which is long-term sequelae $(6,8)$.

Carez and Winkler (8) found that infants born with umbilical artery $\mathrm{pH}$ of less than 7.20 were at greater risk of short-term neonatal complications attributable to intrapartum hypoxia. Severe acidemia of $\mathrm{pH} 7.00$ or less with metabolic acidosis as well as low Apgar score (-3) increased the risk of neonatal complications of the type commonly associated with neurological sequel including mortality. The response time is recorded because the period of exposure of the foetus in utero to foetal distress is one factor that will contribute to immediate perinatal outcome (6-8). However, it is also noted that cerebral damage may begin before onset of labour if the foetus was subjected to hypoxia. In a study of obstetric management of foetal distress and its association with cerebral palsy, Richmond concluded that intrapartum asphyxia is probably more damaging once superimposed on underlying hypoxia (3).

Being among the top three indications for emergency Caesarian section at Kenyatta National Hospital, the prevalence of foetal distress at Kenyatta National Hospital was found to be $5.2 \%$ in the Nairobi birth survey (9). However, clinical diagnosis of foetal distress is not always followed by evidence of newborn asphyxia and a number of babies are born with a good Apgar score of 7 out of ten or more at one minute. Such scores cast some doubt on the validity of the clinical diagnosis of foetal distress and hence the indication for emergency Caesarian section.

In Kenyatta National Hospital, women are usually delivered abdominally if foetal distress is diagnosed before second stage of labour or when second stage is not imminent. The Caesarian section rate has steadily increased from an average of $18 \%$ from 1977 to 1983 (10) to an average of about $20 \%$ from 1984 to 1989 (11) and currently stands at approximately $29 \%$ (12). The trend roughly corresponds to those seen in other countries throughout the world.

Foetal distress is among the top three indications for emergency Caesarean section in Kenyatta National Hospital, yet most newborns delivered have a good Apgar score at one and five minutes. A few show poor Apgar score and signs of asphyxia necessitating admission in special care baby unit. The parameters often used to diagnose foetal distress are: foetal heart irregularity with either tachycardia of more than 160 beats per minute or bradycardia of less than 110 beats per minute determined using Pinnard's foetoscope and meconeum staining liquor, especially grade 2 and above. No study has been carried out in our set up linking clinical foetal distress and perinatal outcome. The objective of the study was to determine the relationship between clinical diagnosis of foetal distress and early perinatal outcome. 


\section{MATERIALS AND METHODS}

Study design: This was a prospective study in which early perinatal outcome of a group of newborns delivered through Caesarian section due to clinical foetal distress (on the bases of abnormal foetal heart rate and rhythm by intermittent auscultation, together with meconeum staining of amniotic fluid) in labour was compared with a group of newborns similarly delivered via Caesarian section without a diagnosis of clinical of foetal distress. The outcomes of interest were Apgar score at one and five minutes, umbilical cord blood $\mathrm{pH}$, admissions to the newborn unit, morbidity and mortality within 24 hours post natal.

Study area: The study area was labour ward and its theatre, Newborn Unit and intensive care unit laboratory of Kenyatta National Hospital. The hospital caters for a large number of medical specialties and sub-specialties, the Departmemt of Obstetrics and Gynaecology being one such specialty.

Study population: The study population comprised women admitted at Kenyatta National Hospital

\section{Inclusion criteria: (i) Caesarian section delivery; (ii)} Term gestation (at 37 weeks or above); (iii) Cephalic presentation. (iv) Live birth. (v) Singleton pregnancies. (vi) Fresh still births (foetal heart present at time of recruitment into the study but baby delivered dead).

Exclusion criteria: All patients with a diagnosis of intra-uterine foetal death (IUED), multiple gestation, low birth weight (less than 2500 grams), breech presentation, pre-term deliveries (less than 36 completed weeks), gross foetal abnormalities and all patients who refused to take part in the study.

\section{Sample size determination: Using EPI INFO version 5} software for epidemiology and disease surveillance 1990, the sample size calculation was determined thus:- Prevalence $(\mathrm{P})$ of foetal distress at Kenyatta National Hospital = 5.2\% (9). Confidence level 95\%; Power $=80 \%$; ratio of exposed to non-exposed $=1: 1$; expected percentage of acidemia in newborns delivered without clinically foetal distress (Non-exposed group) $=5 \%$; Expected percentage of acidemia in newborns delivered with clinical foetal distress (Exposed group) $=25 \%$; Sample size $=58$ exposed and 58 non-exposed giving a total of 116.

For each of the 58 mothers in labour awaiting Caesarian section with a clinical diagnosis of foetal distress during the study period, one mother awaiting Caesarian section without clinical foetal distress in labour was selected.

Management of patients: During the study period the patients were managed by interns, registrars (postgraduates), senior registrars and consultants in the obstetric and gynaecology department together with their counter parts in the neonatal unit. A registrar from the paediatric department who was on duty at new-born unit attended to all the newborns delivered via Caesarian section and did the Apgar scoring at one and five minutes.

Procedure: At delivery, the surgeon or his assistant double clamped a $20-\mathrm{cm}$ length of umbilical cord before the infant took the first breath. A blood sample from the umbilical artery of the clamped section was drawn using a needle with heparinised syringe. The drawn sample of the cord blood was taken to intensive care unit laboratory for $\mathrm{pH}$ and gases analysis within 30 minutes of being taken. During a study on the effect of room temperature and time from delivery on cord blood $\mathrm{pH}$ and $\mathrm{pCO}_{2}$ Strickland (13) reported that samples of cord blood drawn can be kept at room temperature in plastic syringes and determination of $\mathrm{pH}$ and $\mathrm{pCO}_{2}$ within 30 minutes without significant alteration in these values i.e. $\mathrm{pH}$ decrease not greater than 0.04 (P-value less than 0.05). For this reason the blood sample were analysed within 30 minutes of collection.

The time when the baby was extracted was noted. The Apgar score at 1 minute and 5 minutes after delivery of the baby was determined and recorded by the neonatologist or midwife receiving the baby to resuscitate. The neonate was followed for the next two days and the outcome recorded. For the purpose of the study the neonates who were admitted into newborn unit and retained for more than 24 hours had the reason for admission recorded. For example, signs of central nervous system irritability such /as increased tone, fisting, and starring, high pitched cry, convulsions, poor Apgar score, respiratory distress and birth asphyxia were all recorded. Any mortality was also recorded. The cost of blood gas analysis and heparin was covered by the research fund. Permission to use intensive 
care unit laboratories was granted by the head of that sub-unit. The neonates were discharged from the study after two days postnatal.

Data collection: The data were recorded in a coded data sheet to facilitate computer analysis. The proportion of Caesarian section contributed by clinical foetal distress was computed from labour ward records of the three years proceeding the study period.

Data processing and analysis: The data were analysed using appropriate standard statistical methods. Significant differences between the groups were assessed using chi-square $\left(X^{2}\right)$ test. A P-value of 0.05 or less signified significant differences between the groups. Measures of association used were the odds ratio and relative risks and their corresponding $95 \%$ confidence intervals.

Ethical considerations: Approval was obtained from both Kenyatta National Hospital Ethical Committee and Kenyatta National Hospital Administration and the information obtained was used strictly for the study purpose and was not used to incriminate or harm anybody. Each mother was explained the nature of the study and written consent obtained.

Study limitations: Sampling of blood from umbilical cord was not entirely easy and was associated with some risks. The umbilical vessels were covered by Wharton's jelly, which was slippery and friable. Great caution was exercised to prevent accidental needle pricks. The non-exposed group of mothers comprised of mothers who underwent Caesarean section for indications other than clinical diagnosis of foetal distress. These other indications may on their own be associated with newborn asphyxia and hence underestimate the relationship between clinical diagnosis of foetal distress and newborn asphyxia. The determination of Apgar score by different health care providers has the potential to introduce inter-observer bias.

\section{RESULTS}

Between January 1999 and December 2001 a total of 8,825 Caesarian sections were performed at Kenyatta National Hospital labour ward theatre. Clinical foetal distress was the indication for Caesarian section in 1,930 cases, which represented about $22 \%$ of Caesarian sections. Hence about one fifth of Caesarian sections performed at Kenyatta National Hospital was due to clinical foetal distress. Most mothers $(88 \%)$ did not have any disease which included hypertensive disease, diabetes mellitus, cardiac disease and anaemia among others. About $3.4 \%$ of mothers with foetal distress had preeclamptic toxaemia together with foetal distress. Close to $76 \%$ of mothers without foetal distress did not have maternal disease, while $13.8 \%$ had preeclampsia. The risk of foetal distress in mothers with maternal disease was not statistically different from those without maternal disease $(R R=0.4 ; 95 \%$ C.I $=0.2-1.2 ; P$-value $=0.09)$. Obstetric complications for which Caesarean section was an indication included antepartum haemorrhage, cephalopelvic disproportion, previous Caesarean section and premature rupture of membranes. About $59 \%$ of mothers with foetal distress had no obstetric complications while $8.6 \%$ of those without foetal distress had no obstetric complications. The complications in those with foetal distress were cephalo-pelvic disproportion in $22.4 \%$ and premature rapture of membranes at term in $5.2 \%$. Among those without foetal distress the complications were previous Caesarian section scars in $38.0 \%$ and cephalo-pelvic disproportion in $29.3 \%$.

About $29 \%$ of neonates with clinical foetal distress had $\mathrm{pH}$ less than 7.10 compared with $3 \%$ in neonates without a diagnosis of clinical foetal distress (Table 1). Again $41 \%$ of neonates with a diagnosis of clinical foetal distress had $\mathrm{pH}$ between 7.10 and 7.20 compared to $13.8 \%$ in non exposed neonates. About $29 \%$ of exposed neonates had cord blood $\mathrm{pH}$ greater than 7.20 (normal $\mathrm{pH}$ ) compared to about $83 \%$ in non exposed neonates. Thus, among newborn babies delivered through Caesarean section for clinical foetal distress, close to $71 \%$ had umbilical artery acidaemia. The risk of umbilical artery acidemia in newborn babies of mothers with clinical foetal distress was 4.1 that of newborn babies without clinical foetal distress (Table 2). The difference was statistically significant $(R R=4.1 ; 95 \%$ C.I: $2.3-7.4 ; P=0.0000$ ).

At one minute after delivery, close to $12 \%$ of neonates exposed to clinical foetal distress had very poor Apgar score (0-3) while there was none among the non exposed newborns (Table 3 ). About $47 \%$ of exposed newborns had moderately poor Apgar score (4-6) compared to $31 \%$ among those not 
exposed to clinical foetal distress. Overall, $41.4 \%$ of babies in exposed group had a good score at one minute of seven or more compared to $69 \%$ of non exposed babies. While immediate newborn umbilical artery acidaemia occurred in $71 \%$ of newborns of mothers with clinical foetal distress (Table 2), low one minute Apgar score occurred in $59 \%$ of such newborns (Tables 3 and 4 ). The risk of low one minute Apgar score in newborns of mothers with clinical foetal distress was 1.9 times that of newborns of mothers who had no foetal distress (table 4 ) and this increased risk was found to be statistically significant $(\mathrm{RR}=1.9 ; 95 \%$ C.I: 1.2-2.9: $P=0.003$ ).

The study revealed $7 \%$ of babies with clinical foetal distress had very poor Apgar score at five minutes while none of the babies not exposed to foetal distress had very poor Apgar score. About 76\% of babies exposed to foetal distress had good Apgar score (7-10) at five minutes compared to $97 \%$ in non exposed babies. The incidence of low five minute Apgar score was markedly much less (24\%) than at one minute. Neonates delivered with clinical foetal distress were seven times more likely to have poor five minute Apgar score compared to those without clinical foetal distress (Table 5) $(\mathrm{RR}=7.0 ; 95 \% \mathrm{CI}=$ $1.7-29.4 ; \mathrm{P}=0.001$ ). Admission to the newborn unit was not required for $64 \%$ of newborns exposed to clinical foetal distress and $83 \%$ of those without clinical foetal distress (Táble 6). Thirty one percent $(31 \%)$ of babies delivered with clinical foetal distress were admitted to newborn unit for more than 24 hours compared to $17 \%$ of those not exposed. The mortality rate among the exposed newborns was $5 \%$ while there was no death among non exposed babies during the first 24 hours.

\section{Table 1}

Distribution of umbilical artery blood $\mathrm{pH}$ according to clinical foetal distress status

\begin{tabular}{llcccc}
\hline \multicolumn{2}{c}{ Clinical foetal distress } & \multicolumn{3}{c}{$\mathrm{pH}$} & Total \\
& & $<7.10$ & $7.10-7.20$ & $>7.20$ & \\
\hline Yes & No. (\%) & $17(29)$ & $24(41)$ & $17(29)$ & $58(100)$ \\
No & No. (\%) & $2(3)$ & $8(14)$ & $48(83)$ & $58(100)$ \\
\hline
\end{tabular}

$\mathrm{pH}-7.20=$ Abnormal i.e. acidaemia. $\mathrm{pH}>7.20=$ Normal

\section{Table 2}

Relationship between foetal distress and umbilical artery academia

\begin{tabular}{|c|c|c|c|c|c|c|}
\hline \multirow{2}{*}{$\begin{array}{l}\text { Clinical foetal } \\
\text { distress }\end{array}$} & \multicolumn{3}{|c|}{ Umbilical artery acidaemia } & \multirow[t]{2}{*}{ Incidence of acidaemia } & \multirow[t]{2}{*}{ RR (95\% C.I) } & \multirow[t]{2}{*}{ P-value } \\
\hline & Yes & No & $(\%)$ & & & \\
\hline \multirow[t]{2}{*}{ Yes } & 41 & 17 & 71 & & & \\
\hline & & & & $4.1(2.3-7.4)$ & 0.0000 & \\
\hline No & 10 & 48 & 17 & & & \\
\hline
\end{tabular}

Table 3

Distribution of one minute Apgar score according to clinical foetal distress status

\begin{tabular}{|c|c|c|c|c|c|}
\hline \multicolumn{2}{|c|}{ Clinical foetal distress } & \multicolumn{3}{|c|}{ Apgar Score } & \multirow[t]{2}{*}{ Total } \\
\hline & & $0-3$ & $4-6$ & $7-10$ & \\
\hline Yes & No. (\%) & $7(12)$ & $27(47)$ & $24(41)$ & $58(100)$ \\
\hline No & No. (\%) & $0(0)$ & $18(31)$ & $40(69)$ & $58(100)$ \\
\hline Total & & 7 & 45 & 64 & 116 \\
\hline
\end{tabular}


Table 4

Relationship between clinical foetal distress and one minute Apgar score

\begin{tabular}{lccccc}
\hline $\begin{array}{l}\text { Clinical foetal } \\
\text { distress }\end{array}$ & $\begin{array}{c}\text { Low l min. Apgar Score } \\
\text { Yes }\end{array}$ & No & $\begin{array}{c}\text { Incidence of low l minute } \\
\text { Apgar Score } \\
(\%)\end{array}$ & RR (95\% CI) & P-value \\
\hline Yes & 34 & 24 & 59.0 & $1.9(1.2-2.9)$ & 0.003 \\
No & 18 & 40 & 31.0 & & \\
\hline
\end{tabular}

Low Apgar score $=0-6$ from Table 7

Table 5

Relationship between clinical foetal distress and five minutes Apgar score

\begin{tabular}{lccccc}
\hline $\begin{array}{l}\text { Clinical foetal } \\
\text { distress }\end{array}$ & Low 5 min. Apgar Score & $\begin{array}{c}\text { Incidence of low } 5 \\
\text { min. Apgar Score } \\
(\%)\end{array}$ & RR (95\% CI) & P-value \\
\hline Yes & Yes & No & 24.1 & & \\
No & 14 & 44 & 3.4 & $7.0(1.7-29.4)$ & 0.001 \\
\hline
\end{tabular}

Low Apgar score $=0-6$ from Table 9

Table 6

Clinical foetal distress and foetal outcome during the first 24 hours post partum

\begin{tabular}{lccccc}
\hline \multicolumn{2}{l}{ Clinical foetal distress } & Discharged & $\begin{array}{c}\text { Outcome } \\
\text { Admitted for }>24 \text { hrs }\end{array}$ & Died & Total \\
\hline Yes & No. $(\%)$ & $37(64)$ & $18(31)$ & $3(5)$ & $58(100)$ \\
No & No. $(\%)$ & $48(83)$ & $10(17)$ & $0(0)$ & $58(100)$ \\
\hline Total & & 85 & 28 & 3 & 116 \\
\hline
\end{tabular}

Table 7

Distribution of reasons for admission to newborn unit for more than 24 hours

\begin{tabular}{llcccl}
\hline Clinical foetal distress & \multicolumn{3}{c}{ Reason for admission } & Total \\
& & Respiratory distress & Sever asphyxia & Others & \\
\hline Yes & No. $(\%)$ & $15(83)$ & $2(11)$ & $1(6)$ & $18(100)$ \\
No & No. $(\%)$ & $3(30)$ & $1(10)$ & $6(60)$ & $10(100)$ \\
\hline Total & & 18 & 3 & 7 & 28 \\
\hline
\end{tabular}

Table 8

Relationship between clinical foetal distress and morbidity and for mortality

\begin{tabular}{lccccc}
\hline $\begin{array}{l}\text { Clinical foetal } \\
\text { distress }\end{array}$ & $\begin{array}{c}\text { Disease morbidity/ } \\
\text { mortality } \\
\text { No. }\end{array}$ & $\begin{array}{c}\text { Incidence of morbidity/ } \\
\text { mortality } \\
(\%)\end{array}$ & RR (95\% CI) & P-value \\
\hline Yes & 21 & 37 & 36 & & \\
No & 10 & 48 & 17 & $2.1(1.1-4.1)$ & 0.02 \\
\hline
\end{tabular}


Eighty three percent of exposed newborns admitted to new born unit were suffering from respiratory distress and $11 \%$ had severe asphyxia composed to $30 \%$ and $10 \%$ respectively in non exposed newborns (Table 7). The difference in incidence of perinatal morbidity/mortality between the two groups was statistically significant $(R R=2.1 ; 95 \%$ C.I $=1.1-4.1 ; P=0.02)$ (Table 8$)$.

\section{DISCUSSION}

In Kenyatta National Hospital, a diagnosis of intrapartum clinical foetal distress is usually made when meconeum staining of liquor is present together with foetal tachycardia or bradycardia with irregular heart rhythm in labour. This was a prospective study in which the diagnosis of clinical foetal distress was on the bases of abnormal foetal heart rate and rhythm by intermittent foetoscope auscultation, together with meconeum staining of amniotic fluid in labour. The measurement of foetal heart activity is performed most accurately by attaching an electrode directly to the foetal scalp $(14,15)$. This has been the technique used in most clinical trials of electronic foetal monitoring. Intermittent foetoscope auscultation of foetal heart activity and external Doppler ultrasound, though more appropriate for widespread screening, provide less precise data than direct electrocardiogram using a foetal scalp electrode (14). In this study, foetal heart rate patterns were detected using foetoscope auscultation by different health care providers all of them at the level of registrar and above. The evaluation of the newborn for Apgar score was undertaken by different cadres of staff including midwives and registrars from paediatrics.

Over and above the imprecise data from use of intermittent foetoscope auscultation of foetal heart activity, interviewer bias is the greatest threat to the validity of the findings in this study due to use of different health care providers using foetoscope in the labour ward without written down criteria and or protocol for determining abnormal foetal heart activity and use of different health care providers for new born evaluation in the theatre. However, the same interviewers used intermittent auscultation of foetal heart activity in those without a diagnosis of clinical foetal distress. Again, the same health providers evaluated all newborns (irrespective of diagnosis of clinical foetal distress) in theatre using
Apgar score. Hence, use of different health care providers in determining exposure and outcome status in this study most likely resulted in nondifferential (random) misclassification: the proportions of subjects erroneously classified on the basis of abnormal foetal heart rate and rhythm into non-clinical foetal distress group and clinical foetal distress group would be the same. Equally the proportions of newborns given erroneous Apgar score levels in the two groups of exposures are likely to have been approximately equal. The effect of this non-differential misclassification would have resulted in diluting any association between clinical foetal distress and newborn acidaemia and low Apgar score in this study.

At the same time, meconeum stained liquor, without categorisation into thin and thick/ particulate meconium (16), was additionally used in the diagnosis of clinical foetal distress. This further assisted in the determining babies born through Caesarean section due to clinical foetal distress and those born without this diagnosis.

Clinical foetal distress is the indication for emergency Caesarian section in about one fifth of Caesarian deliveries $(1,4,9,17)$. This study found clinical foetal distress to be responsible for close to $22 \%$ of Caesarian section deliveries at Kenyatta National Hospital. With the current Caesarian section rate of approximately $28 \%$, the incidence of foetal distress at Kenyatta National Hospital works out to be approximately $6 \%$ of all deliveries. Foetal distress is usually the main indication for Caesarian section, but there may be superimposed maternal disease or other obstetric complications. This study found maternal diseases such as hypertensive disease in pregnancy, uiabetes mellitus, cardiac disease and anaemia to superimpose about $12 \%$ of foetal distress cases. Obstetric complications were present in about $41 \%$ of foetal distress cases.

The principal screening technique for foetal distress and hypoxia during labour is the measurement of foetal heart rate (14). Abnormal decelerations in foetal heart rate and decreased beatto-beat variability during uterine contractions are considered to be suggestive of foetal distress. The detection of these patterns during monitoring by auscultation or during electronic monitoring (cardiotocography) increases the likelihood that the foetus is in distress, but the patterns are not in diagnostic. 
In addition, normal or equivocal heart rate patterns do not exclude the diagnosis of foetal distress. Precise information on the validity of studies is lacking due to the absence of a universally accepted definition of foetal distress (14). For many years acidosis and hypoxemia as determined by foetal scalp $\mathrm{pH}$ were used for this purpose in research and clinical practice, but it is now clear that neither finding is diagnostic of foetal distress (14).

Thus, not all neonates with clinical foetal distress show overt foetal acidaemia because sporadic or intermittent cord compression and smaller degree of trans-placental gaseous exchange may allow for spontaneous physiological corrections of blood $\mathrm{pH}$. Gilstrap et al (18) found umbilical cord blood acidemia in up to $40 \%$ of neonates with foetal bradycardia of less than 110 beats per minute, but acidemia was about $18 \%$ in those with tachycardia. This supports the theory that foetal tachycardia is a sign of good central nervous system response to hypoxia, whereas bradycardia with late decelerations signify compromised foetal brain stem $(13,19,20)$. This study similarly found acidaemia in $71 \%$ of newborns with clinical foetal distress. Newborns with clinical foetal distress were about four times likely to develop newborn acidaemia compared to those without antecedent clinical foetal distress.

Apgar score at one and five minutes tend to correlate poorly with either the cause or outcome, therefore these scores alone should not be considered as either evidence of or consequence of subsequent asphyxia. Although low scores may be evidence of hypoxia, they are influenced by other factors which affect the infant's responsiveness, tone and respiration such as anaesthetic drugs and analgesics given during labour and delivery by Caesarean section. Nevertheless, low Apgar scores at one and five minutes are excellent indicators for identifying infants needing resuscitation. Various studies report low one minute Apgar scores in up to $48 \%$ of newborns with foetal acidaemia and about $16 \%$ at five minutes $(2,8,21,22)$. This study found the proportion of newborns delivered with clinical foetal distress who showed evidence of low Apgar score at one minute to be $59 \%$ and $24.1 \%$ at five minutes. The risk of low Apgar score in newborns with a diagnosis of clinical foetal distress at one minute and five minutes was 2.5 and 7.1 times respectively that of babies without a diagnosis of clinical foetal distress.
When foetal distress occurs in the presence of meconeum staining of amniotic fluid the chances of newborn respiratory depression and mortality are greater than when the meconeum is not present. Perinatal mortality rates have been reported from $8 \%$ with thin meconeum staining of liquor amnii plus abnormal foetal heart rate to $32 \%$ with thick meconeum stained liquor with or without irregular foetal heart rate $(1,23)$. Locally, Fongoh (19) found close to $4 \%$ early perinatal mortality among newborns delivered via Caesarian section at Kenyatta National Hospital in 1984 due to foetal distress evidenced by meconeum staining of liquor and abnormal foetal heart rate. This study found an early perinatal mortality rate of $5 \%$ among neonates exposed to clinical foetal distress in contrast to no mortality among the newborns not exposed to foetal distress. There was increased risk of perinatal morbidity and mortality among newborns with clinical foetal distress compared to those without foetal distress $(R R=2.1 ; 95 \% C I=1.1-4.1 ; P$-value $=0.02$ ).

In conclusion, clinical foetal distress was responsible for about one fifth of Caesarian deliveries of Kenyatta National Hospital. It was associated with increased incidence (71\%) of newborn acidaemia, low one minute and five minute Apgar scores (59\% and $24 \%$ respectively) compared with absence of clinical foetal distress $(17 \%, 31 \%$, and $3 \%$ newborn acidaemia, low one minute and five minute Apgar score respectively). Early perinatal mortality occurred in about $5 \%$ of newborn babies of mothers who underwent Caesarean section with a diagnosis of clinical foetal distress while among none exposed there was no early perinatal death. The relative risk of poor perinatal outcome in terms of foetal acidaemia, low Apgar score, early perinatal morbidity and or mortality in newborns with clinical foetal distress was at least twice that of newborns without foetal distress. Therefore, meconeum staining of liquor present together with abnormal foetal heart rate and rhythm are still good indicators of foetal jeopardy, which require expedient delivery.

\section{ACKNOWLEDGEMENT}

To the consultants and nursing staff of the Department of Obstetrics and Gynaecology of Kenyatta National Hospital for allowing their patients to be recruited into this study. 


\section{REFERENCES}

1. Miller F., Sacks D., Paul R. et al. Significance of meconeum staining of liquor during labour. Amer. J. Obstet. Gynaecol. 1975; 122: 573-580.

2. Sykes C.S. Do Apgar scores indicate asphyxia? The Lancet. 1982; 1: 494-496.

3. Richmond S., Niswander K., Snodgrass C.A. and Wagstaff I. The obstetric management of foetal distress and its association with cerebral palsy. J. Obstet. Gynaecol. 1994; 83: 643-646.

4. Low J.A., Cox M.J., Karchmer E.J. et al. The prediction of intrapartum foetal metabolic acidosis by foetal heart rate monitoring. Amer. J. Obstet. Gynaecol. 1981; 139: 299-305.

5. Manning F.A., Basket T.F. and Haris F. Foetal biophysical profile scoring. Amer. J. Obstet. Gynaecol. 1981; 140: 289-294.

6. Beard R.W. and Rivers R.P.A. Foetal asphyxia in labour. Lancet. 1979; 2: 1117-1119.

7. Starks G.C. Correlation of meconeum stained amniotic fluid, early interpretations of foetal blood $\mathrm{pH}$ and Apgar scores as predictors of perinatal outcome. Obstet. Gynaecol. 1980; 56: 604-609.

8. Carey L,. Winkler M.D. and John C. Neonatal complications as related to degree of umbilical artery acidemia. Amer. J. Obstet. Gynaecol. 1999; 164: 637-641.

9. Mati J.K.G., Aggarwal N.P. and Sanghvi H.C.G. Nairobi birth survey III: Labour and Delivery J. Obstet. Gynnecol. East and Central Africa. 1983; 2: 47-56.

10. Kenyatta National Hospital Labour Ward Statistics Records, 1977-1984.

11. Muriu F.K.J. A retrospective study on Caesarian section pattern at Kenyatta National Hospital. MMed Thesis University of Nairobi, 1991.

12. Kenyatta National Hospital Labour Ward Statistics Records, 2005.
13. Strickland D.M. Umbilical cord $\mathrm{pH}$ and $\mathrm{pCO}_{2}$ effect of interval from delivery to analysis. Amer.J. Obstet. Gynaecol. 1984; 148: 191-194.

14. Clinical preventive services, second edition. Prenatal disorders: Intrapartum electronic foetal monitoring; hhtp:/ cpmcnet.columbia.edu/texts/gcps/gcps 0049.html 2000.

15. Macdonald D., Grant A., Sheridan-Pereira M. et al. The Dublin randomised controlled trial of intrapartum foetal heart rate monitoring. Amer. J. Obstet. Gynaecol. 1985; 1: 524-539.

16. Klinger M.C. and Kruse J. Meconeum aspiration syndrome: Pathophysiology and prevention. J. Amer. Board Fam. Pract. 1999; 12: 450-466.

17. Birgit D., Seebach M., Gobal D. et al. Prediction of foetal acidosis by means of intrapartum foetal pulse oxymetry. Amer. J. Obstet. Gynaecol. 1999; 180: 73-81.

18. Gilstrap J.C., Hauth J.C., Beck A.W. et al. Foetal heart rate abnormalities and neonatal acidosis. Obstet. Gynaecol. 1987; 70: 191-195.

19. Fongoh F.B. Perinatal mortality at Kenyatta National Hospital contributed by meconeum staining of anmniotic fluid; MMed dissertation (obstetric long case), University of Nairobi, 1984.

20. Hon E.H. and Patrice R.H. Clinical value of foetal heart rate monitoring. Clinics Obstet. Gynecol. 1975; 18: 1-23.

21. Moses B., Horn P., Migue S., et al. Inhibition of pulmonary surfactant formation by meconeum. Amer. J. Obstet. Gynaecol. 1991; 164: 477-481.

22. Karl S., William P., Murphy et al. Criteria to allocate infants to severe asphyxia group. Brit. J. Obstet. Gynaecol. 1990; 97: 470-479.

22. Resnick L. Foetal distress: a complication of foetal mortality in relation to meconeum staining of liquor amnii and post maturity. South Afr. Med. J. 1955; 29: 857-863. 